\title{
Cronin effect and energy conservation constraints in high energy proton-nucleus collisions
}

\author{
Enrico Cattaruzza* and Daniele Treleani ${ }^{\dagger}$ \\ Dipartimento di Fisica Teorica dell'Università di Trieste and INFN, Sezione di Trieste, \\ Strada Costiera 11, Miramare-Grignano, I-34014 Trieste,Italy.
}

(Dated: 28th November 2018)

\begin{abstract}
We estimate the Cronin effect in $p A$ collisions at the CERN LHC and at RHIC, using a Glauber-Eikonal model of initial state multiparton interactions. For a correct determination of the initial parton flux, we upgrade the model cross section, taking carefully into account all kinematical constraints of each multi-parton interaction process. As compared with previous results, derived with approximate kinematics, we obtain a softer spectrum of produced partons, while improving the agreement of the model with the recent measurements of $\pi^{0}$ production in $d+A u$ collisions at $\sqrt{s}=200 \mathrm{AGeV}$.

PACS numbers: $24.85+\mathrm{p}, 11.80 . \mathrm{La}, 25.75 .-\mathrm{q}$
\end{abstract}

Keywords: Hadron-nucleus Collisions, Multiple Scattering

\section{INTRODUCTION}

Hadron-nucleus interactions represent an intermediate regime between hadron-hadron and nucleus-nucleus collisions, where different ideas on the role played by the complexity of the target on large $p_{t}$ dynamics may be tested [1]. Of particular interest is the study of the Cronin effect $[2,3]$, namely the deformation of the transverse spectrum of particles, or jets, produced in hadron-nucleus collisions in the projectile fragmentation region. Given the large scale of momentum transfer in the process, the problem is within reach of a perturbative approach, while the effects of the complex structure of the target may be controlled by changing energy and atomic mass number.

The effect is originated by the high density of the nuclear target, which induces the projectile's partons interacting simultaneously with several partons of the target. There is not however a unique explanation of the underlying dynamical mechanism [4-8] and different approaches can only be discriminated by measuring the large $p_{t}$ spectra in hadron nucleus collisions at the energies of the future colliders, where various models give different predictions. In our opinion, the simplest approach is to adopt the Glauber prescription of factorization of the overall manyparton $S$ matrix, as a product of elementary partonic $S$ matrices [9]. Since production at the

${ }^{*}$ Electronic address: ecattar@ts.infn.it

${ }^{\dagger}$ Electronic address: daniel@ts.infn.it 
parton level represents a higher order correction in the coupling constant, the natural zeroth order approximation is obtained when evaluating each elementary parton interaction at the lowest order in $\alpha_{S}$, which leads considering binary processes only.

Encouragingly a recent calculation, based on this simplest scheme [10], compares rather reasonably with the latest experimental measurements of the Cronin effect in large $p_{t} \pi^{0}$ production in $d+A u$ collisions, at a center of mass energy of $200 \mathrm{AGeV}$ [11], [12], [13], [14], which represents a convenient channel to study the effect (as, differently from charged particle production, there is no contamination from protons, whose production mechanism poses additional problems).

It's remarkable that in such an approach, when adopting the usual approximations of the Glauber picture of high energy hadron-nucleus collisions, the whole effect of the nuclear target medium may be taken into account exactly, in the evaluation of the inclusive transverse spectrum [9]. After summing all possible multi-parton interactions of the projectile parton one in fact obtains

$$
\frac{d \sigma}{d^{2} b d x d^{2} p_{t}}=\frac{1}{(2 \pi)^{2}} \int d^{2} r e^{i p_{t} r} G(x) S_{\text {hard }}^{A}\left(\bar{r}, \bar{b}, p_{0}\right)
$$

where

$$
S_{\text {hard }}^{A}\left(\bar{r}, \bar{b}, p_{0}\right)=\left[e^{T_{A}(b)\left[F_{A}(x, r)-F_{A}(x, 0)\right]}-e^{-T_{A}(b) F_{A}(x, 0)}\right]
$$

and $T_{A}(b)$ is the usual nuclear thickness, as a function of the hadron-nucleus impact parameter $b, G(x)$ the parton number density of the projectile, as a function of the fractional momentum $x, p_{t}$ the transverse momentum of the final observed parton and

$$
F_{A}(x, b)=\int \frac{d^{2} p_{t}}{(2 \pi)^{2}} d x^{\prime} G_{A}\left(x^{\prime}\right) \frac{d \hat{\sigma}}{d^{2} p_{t}} e^{i p_{t} b}
$$

In Eq.(2) $G_{A}\left(x^{\prime}\right)$ is the parton number density of the target nucleus, divided by the atomic mass number $A$, as a function of the fractional momentum $x^{\prime}$, and $d \hat{\sigma} / d^{2} p_{t}$ the elementary partonparton scattering cross section, which includes also the kinematical limits constraints. For sake of simplicity flavor dependence is not written explicitly. The quantity $S_{\text {hard }}^{A}\left(\bar{r}, \bar{b}, p_{0}\right)$ can be interpreted in terms of dipole-nucleus hard cross section. The dipole originates from the square of the scattering amplitude, expressed as a function of the Fourier variable $\bar{r}$, corresponding to the transverse size of the dipole [15].

By expanding Eq.(1) as a power series in $\hat{\sigma}$, one obtains all different multiple scatterings of the projectile parton. As a consequence of the Glauber prescription of factorization of the overall many-parton $S$ matrix, each term in the expansion is represented by an incoherent convolution 
of binary partonic collisions $[9,16]$. Analogously to the case of the Glauber approach to hadronnucleus processes, the $S$-matrix factorization prescription does not however imply any spacetime ordering between the different elementary collisions. It only implies that a connected $n$-body interaction process is well approximated by a product of two-body interactions, which corresponds to the dominance of the pole contribution, in a dispersive representation of the projectile-exchanged gluon amplitude [17].

All expressions are divergent for small values of the lower cutoff in $p_{t}$, which sets the regime of hard interactions. Nevertheless, as unitarity is explicitly implemented, each term in the series expansion of Eq.(1) contains subtractions, which lower the degree of the infrared singularity, from an inverse power to a power of a logarithm of the cutoff [16]. It's also worth mentioning that, in this model, the average energy, carried by the partons scattered to the final state is bound to be smaller than the overall initial state energy [18], whereas such a quantity is divergent as an inverse power of the cutoff, if evaluated in impulse approximation.

The approach to hard processes in hadron-nucleus collisions, based on the factorization of the multi-parton $S$ matrix, is hence unitary and satisfies various non-trivial consistency requirements, including the AGK cutting rules [19]. As $p_{t} \rightarrow 0$ the importance of unitarity corrections grows, producing a suppression of the integrated parton yield and a random walk of partons to higher $p_{t}$, recovering in this way the local isotropy in transverse space of the black disk limit, which is, on the contrary, maximally broken by the lowest order impulse approximation term [18].

The model hence contains geometrical shadowing at low $p_{t}$ and Cronin enhancement of the transverse spectrum at moderate $p_{t}$. Some non secondary features of the interaction are not accounted however, as one expects genuine dynamical shadowing at low $x$, due to non-linear gluon interactions [1]. In spite of some interesting efforts in this direction [20], incorporating non-linear gluon evolution in the present formalism is however a non trivial problem, which we will not try to address in the present note.

In fact we aim to point out that also some of the simplifying assumptions, adopted in the derivation of Eq.(1), require a closer inspection. More specifically, to obtain Eq.(1), one needs to decouple longitudinal and transverse degrees of freedom, while conserving the longitudinal momentum components in the interaction. Kinematical constraints are hence implemented only approximately, in such a way that final state partons turn out to be more energetic than the initial ones, the effect being emphasized when the number of re-scatterings grows. The final $p_{t}$ spectrum gets hence shifted towards larger transverse momenta which, given the steepness of parton distributions at small $x$, might modify appreciably the evaluation of the Cronin effect.

The purpose of the present note is to investigate this specific aspect of the model more closely. We hence compare the spectrum, obtained by implementing exactly all kinematical constraints, with the summed expression in Eq.(1), both at the LHC regime and in the kinematical domain of the recent measurements of the Cronin effect in $d+A u \rightarrow \pi^{0} X$ at RHIC. 


\section{TRANSVERSE SPECTRUM}

The expression in Eq.(1) represents the sum of all possible collisions of the observed parton with the different scattering centers of the target nucleus. When the kinematical constraints are implemented exactly, the multi-scattering series cannot be re-summed any more in a closed analytic expression and each different term needs to be evaluated separately. Most of the spectrum is nevertheless well reproduced by the the first three terms of the expansion [16]. Hence we approximate the cross section by:

$$
\frac{d \sigma}{d^{2} p_{t} d y d^{2} b}=\sum_{i} \frac{d \sigma_{i}}{d^{2} p_{t} d y d^{2} b}
$$

where $p_{t}$ is the transverse momentum of the observed parton, $y$ its rapidity, $b$ the impact parameter of the collision and the sum runs over the different species of projectile partons, while

$$
\frac{d \sigma_{i}}{d^{2} p_{t} d y d^{2} b}=\frac{d \sigma_{i}^{(1)}}{d^{2} p_{t} d y d^{2} b}+\frac{d \sigma_{i}^{(2)}}{d^{2} p_{t} d y d^{2} b}+\frac{d \sigma_{i}^{(3)}}{d^{2} p_{t} d y d^{2} b}
$$

where

$$
\begin{aligned}
\frac{d \sigma_{i}^{(1)}}{d^{2} p_{t} d y d^{2} b} & =T_{A}(b) \sum_{j} \frac{1}{1+\delta_{i j}} \int d^{2} q_{1} d x^{\prime} \delta^{(2)}\left(\bar{q}_{1}-\bar{p}_{t}\right) \hat{\sigma_{i j}}{ }^{(1)}\left(y, x^{\prime} ; q_{1}\right) \\
& \times x\left[f_{\frac{i}{p}}\left(x, Q_{f c t}\right) f_{\frac{j}{A}}\left(x^{\prime}, Q_{f c t}\right)+f_{\frac{i}{p}}\left(x^{\prime}, Q_{f c t}\right) f_{\frac{j}{A}}\left(x, Q_{f c t}\right)\right]
\end{aligned}
$$

is the single scattering term (the projectile parton interacts with a single parton of the target and vice-versa),

$$
\begin{aligned}
\frac{d \sigma_{i}^{(2)}}{d^{2} p_{t} d y d^{2} b} & =\frac{1}{2 !} T_{A}(b)^{2} \sum_{j, k} \frac{1}{1+\delta_{i j}} \frac{1}{1+\delta_{i k}} \int d^{2} q_{1} d^{2} q_{2} d x^{\prime} d x^{\prime \prime} \\
& \times\left[\delta^{(2)}\left(\bar{q}_{1}+\bar{q}_{2}-\bar{p}_{t}\right)-\delta^{(2)}\left(\bar{q}_{1}-\bar{p}_{t}\right)-\delta^{(2)}\left(\bar{q}_{2}-\bar{p}_{t}\right)\right] \\
& \times x f_{\frac{i}{p}}\left(x, Q_{f c t}\right) f_{\frac{j}{A}}\left(x^{\prime}, Q_{f c t}\right) f_{\frac{k}{A}}\left(x^{\prime \prime}, Q_{f c t}\right) \hat{\sigma}_{i j k}^{(2)}\left(y, x^{\prime}, x^{\prime \prime} ; \bar{q}_{1}, \bar{q}_{2}\right)
\end{aligned}
$$

is the rescattering term (the projectile parton interacts with two different partons of the target), and 


$$
\begin{aligned}
\frac{d \sigma_{i}^{(3)}}{d^{2} p_{t} d y d^{2} b}= & \frac{1}{3 !} T_{A}(b)^{3} \sum_{j, k, l} \frac{1}{1+\delta_{i j}} \frac{1}{1+\delta_{i k}} \frac{1}{1+\delta_{i l}} \int d^{2} q_{1} d^{2} q_{2} d^{2} q_{3} \\
\times & d x^{\prime} d x^{\prime \prime} d x^{\prime \prime \prime} \hat{\sigma}_{i j k l}^{(3)}\left(y, x^{\prime}, x^{\prime \prime}, x^{\prime \prime \prime} ; \bar{q}_{1}, \bar{q}_{2}, \bar{q}_{3}\right) \\
\times & {\left[\delta^{(2)}\left(\bar{q}_{1}+\bar{q}_{2}+\bar{q}_{3}-\bar{p}_{t}\right)-\delta^{(2)}\left(\bar{q}_{1}+\bar{q}_{2}-\bar{p}_{t}\right)-\delta^{(2)}\left(\bar{q}_{2}+\bar{q}_{3}-\bar{p}_{t}\right)\right.} \\
& \left.-\delta^{(2)}\left(\bar{q}_{3}+\bar{q}_{1}-\bar{p}_{t}\right)+\delta^{(2)}\left(\bar{q}_{1}-\bar{p}_{t}\right)+\delta^{(2)}\left(\bar{q}_{2}-\bar{p}_{t}\right)+\delta^{(2)}\left(\bar{q}_{3}-\bar{p}_{t}\right)\right] \\
\times & x f_{\frac{i}{p}}\left(x, Q_{f c t}\right) f_{\frac{j}{A}}\left(x^{\prime}, Q_{f c t}\right) f_{\frac{k}{A}}\left(x^{\prime \prime}, Q_{f c t}\right) f_{\frac{l}{A}}\left(x^{\prime \prime \prime}, Q_{f c t}\right) .
\end{aligned}
$$

is the double rescattering term (the projectile parton interacts with three different partons of the target).

The subtraction terms, in the rescattering and in the double rescattering terms, are a direct consequence of the implementation of unitarity in the process. The sums run over the different species of target partons. The quantities $f_{\frac{j}{A}}\left(x^{\prime}, Q_{f c t}\right)$ represent the nuclear parton distributions, while the multiparton interaction cross sections are given by:

$$
\begin{gathered}
\hat{\sigma}_{i j}^{(1)}\left(y, x^{\prime} ; q_{1}\right)=k_{\text {factor }} \frac{d \hat{\sigma}_{i j}\left(\hat{s}_{1}, \hat{t}_{1}, \hat{u}_{1}\right)}{d \hat{t}_{1}} \Theta\left(\Phi^{(1)}\right) \\
\hat{\sigma}_{i j k}^{(2)}\left(y, x^{\prime}, x^{\prime \prime} ; \bar{q}_{1}, \bar{q}_{2}\right)=\left(k_{\text {factor }}\right)^{2} \frac{d \hat{\sigma}_{i k}\left(\hat{s}_{2}, \hat{t}_{2}, \hat{u}_{2}\right)}{d \hat{t}_{2}} \frac{d \hat{\sigma}_{i j}\left(\hat{s}_{1}, \hat{t}_{1}, \hat{u}_{1}\right)}{d \hat{t}_{1}} \Theta\left(\Phi^{(2)}\right) \Theta\left(\Phi^{(1)}\right) \\
\hat{\sigma}_{i j k l}^{(3)}\left(y, x^{\prime}, x^{\prime \prime}, x^{\prime \prime \prime} ; \bar{q}_{1}, \bar{q}_{2}, \bar{q}_{3}\right) \\
=\left(k_{f a c t o r}\right)^{3} \frac{d \hat{\sigma}_{i l}\left(\hat{s}_{3}, \hat{t}_{3}, \hat{u}_{3}\right)}{d \hat{t}_{3}} \frac{d \hat{\sigma}_{i k}\left(\hat{s}_{2}, \hat{t}_{2}, \hat{u}_{2}\right)}{d \hat{t}_{2}} \\
\times \frac{d \hat{\sigma}_{i j}\left(\hat{s}_{1}, \hat{t}_{1}, \hat{u}_{1}\right)}{d \hat{t}_{1}} \Theta\left(\Phi^{(3)}\right) \Theta\left(\Phi^{(2)}\right) \Theta\left(\Phi^{(1)}\right),
\end{gathered}
$$

where the parton $_{i}$-parton $_{j}$ differential cross sections $d \hat{\sigma}_{i j} / d \hat{t}$ are evaluated at the lowest order in pQCD by making use of exact kinematics. The kinematical constraints are implemented through the quantities $\Phi^{(i)}$. The expressions of $\Phi^{(i)}$ and of the invariants $\hat{s}_{i}, \hat{t}_{i}, \hat{u}_{i}$ are derived in great detail in the appendix.

The infrared singularity is regularized by introducing in the propagators the mass parameter $p_{0}$, which is a free parameter in the model. Higher order-effects in the elementary interaction are accounted by multiplying the lowest order expressions in $\alpha_{s}$ by the factor $k_{\text {fact }}$.

When evaluating the $\pi^{0}$ spectrum, we let partons fragment independently, neglecting the transverse momentum component generated by the fragmentation process. The relations between hadronic and partonic variables are hence the following [21]: 
$m_{h} \cosh y_{h}=z p_{t} \cosh y \quad m_{h} \sinh y_{h}=q_{t} \sinh y$

where $z=\frac{E_{h}}{E}$, with $E$ the energy of the parton, $y$ its rapidity, $E_{h}$ the energy of the hadron $h, m$ its mass, $y_{h}$ its rapidity, $q_{t}$ its transverse momentum and $m_{h}$ its transverse mass. The inclusive $\pi^{0}$ spectrum is hence given by:

$$
\frac{d \sigma^{\text {frag }}}{d^{2} q_{t} d y_{h} d^{2} b}=\sum_{i} \frac{d \sigma_{i}^{\text {frag }}}{d^{2} q_{t} d y_{h} d^{2} b}
$$

with

$$
\frac{d \sigma_{i}^{f r a g}}{d^{2} q_{t} d y_{h} d^{2} b}=\left.J\left(m_{h}, y_{h}\right) \int \frac{d z}{z^{2}} D_{i \rightarrow h}\left(z, Q_{F}^{2}\right) \frac{d \sigma_{i}}{d^{2} p_{t} d y d^{2} b}\right|_{p_{t}, y}
$$

where

$$
\begin{array}{r}
p_{t}=\frac{q_{t}}{z} J\left(m_{h}, y_{h}\right) \quad y=\arcsin \left(\frac{m_{h} \sinh y}{q_{t}}\right) \\
J\left(m_{h}, y_{h}\right)=\left(1-\frac{m^{2}}{m_{h}^{2} \cosh ^{2} y_{h}}\right)^{-\frac{1}{2}}
\end{array}
$$

and $D_{i \rightarrow h}\left(z, Q_{F}^{2}\right)$ the fragmentation functions, depending on the fragmentation scale $Q_{F}$. The integration region for the hadron energy fraction $z$ is set by the request $p_{t} \geq p_{0}$, with $p_{0}$ the infrared regulator:

$$
\frac{2 m_{h} \cosh y_{h}}{\sqrt{s}} \leq z \leq \min \left[1, \frac{q_{t}}{p_{0}} J\left(m_{h}, y_{h}\right)\right]
$$

\subsection{Numerical Results}

In our calculations we use the isospin averaged nuclear parton distributions, $f_{\frac{i}{A}}=Z f_{\frac{i}{p}}+$ $(A-Z) f_{\frac{i}{n}}$, where for the proton and the neutron distributions, $f_{\frac{i}{p}}$ and $f_{\frac{i}{n}}$, we take the CTEQ5 parametrization at the leading order [22], while for the nuclear thickness function $T_{A}(b)$, which is normalized to one, we use the Wood-Saxon parametrization.

To study the effect at the LHC we consider the case of production of minijets in a forward calorimeter $(\eta \in[2.4,4])$ at two different center of mass energies in the hadron-nucleon c.m. system, $\sqrt{s}=5.5 \mathrm{ATeV}$ and $\sqrt{s}=8.8 \mathrm{ATeV}$, which might be compatible with the acceptances of ATLAS and CMS [23]. Given the small values of $x$ in this kinematical range, we use effective parton distribution functions, with the gluon-gluon cross-section as a dynamical input. We set the factorization and renormalization scale $Q_{f c t}, Q_{r n}$ equal to the regularized transverse mass 
$m_{t}=\sqrt{p_{0}^{2}+p_{t}^{2}}$ and we evaluate the transverse spectrum of jets, in proton-lead collisions, by the expression:

$$
\frac{d W_{p A}}{d^{2} p_{t}}=\frac{1}{y_{\max }-y_{\min }} \int_{y \in\left[y_{\min }, y_{\max }\right]} d x d^{2} b \frac{d \sigma}{d^{2} p_{t} d y d^{2} b}
$$

where the integrand is given by Eq.(3). As for the choice of the infrared regulator we set $p_{0}=2 \mathrm{GeV}$ and we use $k_{\text {factor }}=2$. Our results are plotted in Fig.1, where we compare the spectrum (5) obtained by the exact implementation of energy conservation in the multiple interactions (solid line), with the approximate kinematics results given by the first three terms of the expansion of Eq.(1). As an effect of the exact implementation of kinematics the spectrum of the outgoing particle is shifted towards lower transverse momenta, resulting in an appreciable energy loss effect: at $p_{t} \sim 15 \mathrm{GeV}$ the energy-loss correction to the spectrum is of the order of 40$50 \%$, at higher transverse momenta, $p_{t} \sim 30 \mathrm{GeV}$, it's still about 30-38\%. The triple scattering approximation, used to evaluate the spectrum, breaks down at $p_{t} \leq 9 \mathrm{GeV}$ at $\sqrt{s}=8.8 \mathrm{TeV}$ : by increasing the center of mass energy the density of target partons grows rapidly and the contribution of higher order rescatterings cannot be neglected any more at $p_{t} \leq 9 \mathrm{GeV}$ (right panel of Fig.1). As the energy is lowered to $\sqrt{s}=5.5 \mathrm{TeV}$, the region of numerical instability is shifted to the region $p_{t} \leq 3 \mathrm{GeV}$ (left panel of Fig.1).

The softening of the spectrum has important effects on the Cronin ratio, defined as:

$$
R\left(p_{t}\right)=\frac{d W_{p A} / d^{2} p_{t}}{d W_{p A}^{(1)} / d^{2} p_{t}}
$$

where $d W_{p A}^{(1)} / d^{2} p_{t}$ is the cross section in impulse approximation. The Cronin ratio, corresponding to the spectra in fig.1, is shown in fig.2, where the dashed and continuous lines correspond to approximate and exact kinematics respectively. The two panels of fig. 2 refer to the two values considered for the center of mass energy; at higher energy multiple interactions produce a larger value of the Cronin ratio. The overall effect of the implementation of energy conservation on $R$ is to decrease the size of the Cronin effect and to shift the curve towards smaller transverse momenta.

At lower energy, $\sqrt{s}=200 \mathrm{AGeV}$, we follow [10] in the evaluation of the cross section of $d+A u \rightarrow \pi^{0} X$, using $Q_{f c t}=Q_{r n}=\frac{m_{t}}{2}$, and the values $p_{0}=1.0 \mathrm{GeV}$ and $k_{f a c t}=1.04$. These choices, besides minimizes the effects of higher order corrections in $\alpha_{S}$, allow one reproducing the inclusive cross section of $\pi^{0}$ production in $p p$ collisions at the same c.m. energy, without any need of smearing the cross section with an intrinsic parton transverse momentum. At $\sqrt{s}=200$ $\mathrm{AGeV}$ we take explicitly into account the differences between partons, both in the distributions and in the evaluation of the partonic cross sections. The resulting Cronin ratio in $d+A u$ collisions is hence a parameter free prediction of the our model. By using the leading order 
$K-K-P$ fragmentation functions [24] at the fragmentation scale $Q_{F}=\frac{m_{t}}{2}$, we evaluate:

$$
R_{d A u \rightarrow \pi^{0} X}=\frac{d \sigma_{d A u \rightarrow \pi^{0} X}^{\text {frag }}}{d^{2} q_{t} d y d^{2} b} / \frac{d \sigma_{d A u \rightarrow \pi^{0} X}^{\text {frag }(1)}}{d^{2} q_{t} d y d^{2} b}
$$

at $y=0$ and $b=b_{d A u}=5.7 \mathrm{fm}$, which is the estimated average impact parameter of the experiment [25]. In fig.3 we compare our result (continuous line) with the experimental data of the PHENIX collaboration [11] and with the result obtained by using the approximate kinematics of Eq.(1), dashed line. Given the small rapidity values of the observed $\pi^{0}$, in this case the corrections induced by exact kinematics are particularly important in the region of smaller transverse momenta. The dependence of the effect on the impact parameter is shown in fig.4, where our calculation (continuous line) is compared with preliminary data presented by PHENIX at the DNP Fall Meeting, held in Tucson last October 2003 [26], and with the the standard Glauber-Eikonal calculation (dashed line). The fractions refer to the BBC centrality selection. The corresponding estimated impact parameter values are: $0-20 \%=>b=3.5 \mathrm{fm} 20-40 \% \rightarrow b=4.5 \mathrm{fm} 40-60 \% \rightarrow$ $\mathrm{b}=5.5 \mathrm{fm} 60-88 \% \rightarrow \mathrm{b}=6.5 \mathrm{fm}$. As an effect of implementing exactly kinematical constraints, one obtains a reduction of the Cronin curve in the region of smaller $p_{t}$ values, resulting with an improved agreement of the model with the experimental indication. Notice that besides the deformation of the spectrum leading to geometrical shadowing, by implementing energy conservation one obtains also the quenching the spectrum, as part of the projectile's energy is transferred to the target.

The expectation for $\pi^{0}$ production in $d+A u$ at $\eta=3.2$ is shown in fig.5, where we compare our result (continuous line) with the standard Glauber-Eikonal model calculation (dashed line). Given the larger average number of rescatterings at larger rapidity values, the quenching of the spectrum, due to the energy lost by the projectile in the multiple collisions, is now sizably in-

creased (notice that the vertical scale is now different with respect to the previous figures 3 and 4). One in fact obtains values of $R_{d A u \rightarrow \pi^{0} X}$ as small as .6 at $p_{t}=1 \mathrm{GeV}$. The expectation is nevertheless that the Cronin curve should exceed one for $p_{t} \geq 2 \mathrm{GeV}$, which is not the case for charged particle production. In the figure we plot preliminary results for charged particle production at $\eta=3.2$, presented by BRAHMS at the Quark Matter Conference[27]. In the forward region the quenching of the spectrum might hence be a sizably larger effect, not accountable by multiple scatterings.

\subsection{Conclusions}

In very high energy proton-nucleus collisions the interaction probability is very large, also when considering processes with momentum transfer well above the lower limit for a perturbative approach. In this regime unitarity needs to be implemented already at the level of perturbative interactions and the Glauber-Eikonal model of multiple parton collisions allows implementing 
unitarity in the simplest and most natural way. A standard approximation is to conserve the longitudinal momentum component of the projectile in the process. The approximation becomes however questionable when dealing with parton interactions, since parton distributions are singular at small $x$ and a small change of the parton momentum leads to sizable modifications of the initial parton flux.

In the present note we have studied this particular aspect of the problem, working out the leading terms in the expansion in multiple parton collisions and taking into account all kinematical constraints exactly. Our result is that the spectrum of the produced large $p_{t}$ partons is appreciably softened after implementing exact kinematics.

The Cronin effect has been recently measured in the large $p_{t}$ spectrum of $\pi^{0}$ production in proton-lead collisions, at $y=0$ and $\sqrt{s}=200 \mathrm{AGeV}$. We have worked out the $\pi^{0}$ spectrum in the same kinematical conditions and found a rather good agreement of our model with experimental data. In this case the corrections to the Glauber Eikonal model, induced by implementing kinematics exactly, are increasingly important in the region of smaller $p_{t}$, where, given the rapidity value of the observed $\pi^{0}$, longitudinal and transverse momentum components are of comparable magnitude. The quenching of the spectrum, due to the energy lost by the projectile through multiple collisions, is a stronger effect at larger rapidity. We have estimated the effect at $\eta=3.2$, where the preliminary experimental indications seem however to indicate a stronger quenching effect than expected in the model, which, at relatively large $p_{t}$, still gives a value of the Cronin ratio larger that one.

\section{ACKNOWLEDGMENTS}

We are grateful to M. Tosolini for his valuable computational support. One of us, D.T., thanks the Department of Energy's Institute for Nuclear Theory at the University of Washington for hospitality and the Department of Energy for partial support during the completion of this work. This work was partially supported by the Italian Ministry of University and of Scientific and Technological Researches (MIUR) by the Grant COFIN2003.

\section{APPENDIX}

The four-body parton interaction (one projectile parton against three target partons) is represented in the model by the convolution of three on-shell elastic parton-parton scatterings. We call $h$ the four-momentum of the initial state projectile parton, and $p^{\prime}, p^{\prime \prime}, p^{\prime \prime \prime}$, the four-momenta of the three nuclear target partons. All partons are massless and on shell and have zero transverse momentum components. By introducing the momentum fractions $x, x^{\prime}, x^{\prime \prime}$, the light-cone components of the four momenta in the initial state are: 


$$
h=(\sqrt{s} x, 0 ; \overline{0}) \quad p^{\prime}=\left(0, \sqrt{s} x^{\prime} ; \overline{0}\right) \quad p^{\prime \prime}=\left(0, \sqrt{s} x^{\prime \prime} ; \overline{0}\right) \quad p^{\prime \prime \prime}=\left(0, \sqrt{s} x^{\prime \prime \prime} ; \overline{0}\right)
$$

The transverse components of the exchanged four-momenta are $\bar{q}_{1}, \bar{q}_{2}$ and $\bar{q}_{3}$, while $I, J, K$ are the projectile's four-momenta after the first, second and third interaction and $P, Q$ and $R$ the four-momenta of the recoiling target partons. For the transverse components one has:

$$
I_{\perp}=\bar{q}_{1} \quad J_{\perp}=\bar{q}_{1}+\bar{q}_{2} \quad K_{\perp}=\bar{q}_{1}+\bar{q}_{2}+\bar{q}_{3} \quad P_{\perp}=-\bar{q}_{1} \quad Q_{\perp}=-\bar{q}_{2} \quad R_{\perp}=-\bar{q}_{3}
$$

While the energy conservation and mass-shell conditions, for each two-body scattering process, are expressed by the following equations:

$$
\begin{gathered}
\left\{\begin{array}{cll}
I^{+} I^{-} & = & \bar{q}_{1}^{2} \\
P^{+} P^{-} & = & \bar{q}_{1}^{2} \\
\sqrt{s} x & = & I^{+}+P^{+} \\
\sqrt{s} x^{\prime} & = & I^{-}+P^{-}
\end{array}\right. \\
\left\{\begin{array}{ccc}
J^{+} J^{-} & = & \left.\bar{q}_{1}+\bar{q}_{2}\right)^{2} \\
Q^{+} Q^{-} & = & \bar{q}_{2}^{2} \\
I^{+} & = & J^{+}+Q^{+} \\
I^{-}+\sqrt{s} x^{\prime \prime} & = & J^{-}+Q^{-} \\
& & \\
K^{+} K^{-} & = & \left(\bar{q}_{1}+\bar{q}_{2}+\bar{q}_{3}\right)^{2} \\
R^{+} R^{-} & = & \bar{q}_{3}^{2} \\
J^{+} & = & K^{+}+R^{+} \\
J^{-}+\sqrt{s} x^{\prime \prime \prime} & = & K^{-}+R^{-}
\end{array}\right.
\end{gathered}
$$

The three systems of equations allow to determine

$$
x, I^{+}, I^{-}, J^{+}, J^{-}, K^{-}, P^{+}, P^{-}, Q^{+}, Q^{-}, R^{+}, R^{-}
$$

as a function of $\bar{q}_{1}, \bar{q}_{2}, \bar{q}_{3}, K^{+}, x^{\prime}, x^{\prime \prime}$ and $x^{\prime \prime \prime}$.

From the system (8) and the mass shell condition $J^{+} J^{-}=\left(\bar{q}_{1}+\bar{q}_{2}\right)^{2}$, one obtains:

$$
\begin{aligned}
& \left(\sqrt{s} x^{\prime \prime \prime} K^{+}-\left(\bar{q}_{1}+\bar{q}_{2}+\bar{q}_{3}\right)^{2}\right)\left(J^{+}\right)^{2}-\left(\bar{q}_{1}+\bar{q}_{2}\right)^{2}\left(K^{+}\right)^{2} \\
& \quad-\left(\sqrt{s} x^{\prime \prime \prime} K^{+}-2\left(\bar{q}_{1}+\bar{q}_{2}\right) \cdot\left(\bar{q}_{1}+\bar{q}_{2}+\bar{q}_{3}\right)\right) J^{+} K^{+}=0
\end{aligned}
$$


which has real solutions when $\Lambda^{(3)} \geq 0$, where

$$
\begin{aligned}
\Lambda^{(3)}= & \left(\sqrt{s} x^{\prime \prime \prime} K^{+}\right)^{2}-4\left(\bar{q}_{1}+\bar{q}_{2}\right) \cdot \bar{q}_{3} \sqrt{s} x^{\prime \prime \prime} K^{+} \\
& +4\left(\left(\left(\bar{q}_{1}+\bar{q}_{2}\right) \cdot \bar{q}_{3}\right)^{2}-\left(\bar{q}_{1}+\bar{q}_{2}\right)^{2} \bar{q}_{3}^{2}\right)
\end{aligned}
$$

The condition $\Lambda^{(3)} \geq 0$ can be satisfied only if $\Phi^{(3)} \geq 0$, where:

$$
\Phi^{(3)}=1-2 \frac{\left(\left(\bar{q}_{1}+\bar{q}_{2}\right) \cdot \bar{q}_{3}+\left|\bar{q}_{1}+\bar{q}_{2}\right|\left|\bar{q}_{3}\right|\right)}{\sqrt{s} x^{\prime \prime \prime} K^{+}}
$$

which gives the kinematical limits for $x^{\prime \prime \prime}$ and for $K^{+}$

$$
x_{\text {min }}^{\prime \prime \prime} \equiv \frac{2\left(\left(\bar{q}_{1}+\bar{q}_{2}\right) \cdot \bar{q}_{3}+\left|\bar{q}_{1}+\bar{q}_{2}\right|\left|\bar{q}_{3}\right|\right)}{\sqrt{s} x^{\prime \prime \prime} K^{+}} \equiv \frac{K_{m i n}^{+}}{K^{+}} \leq 1
$$

One hence obtains $J$ and $R$ :

$$
\begin{aligned}
J^{+} & =\frac{K^{+}}{2} \cdot \frac{\sqrt{s} K^{+} x^{\prime \prime \prime}-2\left(\left(\bar{q}_{1}+\bar{q}_{2}\right) \cdot\left(\bar{q}_{1}+\bar{q}_{2}+\bar{q}_{3}\right)\right)+\sqrt{\Lambda^{(3)}}}{\sqrt{s} K^{+} x^{\prime \prime \prime}-\left(\bar{q}_{1}+\bar{q}_{2}+\bar{q}_{3}\right)^{2}} \\
J^{-} & =\frac{\sqrt{\Lambda^{(3)}}+2\left(\left(\bar{q}_{1}+\bar{q}_{2}\right) \cdot\left(\bar{q}_{1}+\bar{q}_{2}+\bar{q}_{3}\right)\right)-\sqrt{s} K^{+} x^{\prime \prime \prime}}{2 K^{+}} \\
R^{+} & =\frac{K^{+}}{2} \cdot \frac{\sqrt{\Lambda^{(3)}}+2\left(\bar{q}_{3} \cdot\left(\bar{q}_{1}+\bar{q}_{2}+\bar{q}_{3}\right)\right)-\sqrt{s} K^{+} x^{\prime \prime \prime}}{\sqrt{s} K^{+} x^{\prime \prime \prime}-\left(\bar{q}_{1}+\bar{q}_{2}+\bar{q}_{3}\right)^{2}} \\
R^{-} & =\frac{\sqrt{\Lambda^{(3)}}+\sqrt{s} K^{+} x^{\prime \prime \prime}-2\left(\bar{q}_{3} \cdot\left(\bar{q}_{1}+\bar{q}_{2}+\bar{q}_{3}\right)\right)}{2 K^{+}},
\end{aligned}
$$

while the Mandelstam invariants $\hat{s}_{3}=\left(J+p^{\prime \prime \prime}\right)^{2}, \hat{t}_{3}=(K-J)^{2}, \hat{u}_{3}=(R-J)^{2}$ are expressed by:

$$
\begin{aligned}
& \hat{s}_{3}=\sqrt{s} x^{\prime \prime \prime} J^{+} \\
& \hat{t}_{3}=-\frac{\left(\left(\bar{q}_{1}+\bar{q}_{2}+\bar{q}_{3}\right) J^{+}-K^{+}\left(\bar{q}_{1}+\bar{q}_{2}\right)\right)^{2}}{K^{+} J^{+}} \\
& \hat{u}_{3}=-\frac{\left(J^{+} \bar{q}_{3}+R^{+}\left(\bar{q}_{1}+\bar{q}_{2}\right)\right)^{2}}{J^{+} R^{+}}
\end{aligned}
$$

After solving (7) with respect to $I$ and $Q$ as a function of $\bar{q}_{1}, \bar{q}_{2}, \bar{q}_{3}, J^{+}$and $x^{\prime \prime}$ and using the mass shell condition $I^{+} I^{-}=\bar{q}_{1}^{2}$ one obtains:

$$
\begin{aligned}
& \left(\sqrt{s} x^{\prime \prime} J^{+}-\left(\bar{q}_{1}+\bar{q}_{2}\right)^{2}\right)\left(I^{+}\right)^{2}-\left(\bar{q}_{1}\right)^{2}\left(J^{+}\right)^{2} \\
& \quad-\left(\sqrt{s} x^{\prime \prime} J^{+}-2 \bar{q}_{1} \cdot\left(\bar{q}_{1}+\bar{q}_{2}\right)\right) I^{+} J^{+}=0
\end{aligned}
$$


which has real solutions when $\Lambda^{(2)} \geq 0$, where

$$
\Lambda^{(2)}=\left(\sqrt{s} x^{\prime \prime} J^{+}\right)^{2}-4 \bar{q}_{1} \cdot \bar{q}_{2} \sqrt{s} x^{\prime \prime} J^{+}+4\left(\left(\bar{q}_{1} \cdot \bar{q}_{2}\right)^{2}-\bar{q}_{1}^{2} \bar{q}_{2}^{2}\right)
$$

The condition $\Lambda^{(2)} \geq 0$ is satisfied only if $\Phi^{(2)} \geq 0$, where

$$
\Phi^{(2)}=1-2 \frac{\left(\bar{q}_{1} \cdot \bar{q}_{2}+\left|\bar{q}_{1}\right|\left|\bar{q}_{2}\right|\right)}{\sqrt{s} x^{\prime \prime} J^{+}}
$$

which gives the kinematical limits for $x^{\prime \prime}$ and for $K^{+}$

$$
x_{\min }^{\prime \prime} \equiv \frac{2\left(\bar{q}_{1} \cdot \bar{q}_{2}+\left|\bar{q}_{1}\right|\left|\bar{q}_{2}\right|\right)}{\sqrt{s} x^{\prime \prime} J^{+}} \equiv \frac{J_{m i n}^{+}}{J^{+}} \leq 1
$$

One hence obtains I and $Q$ :

$$
\begin{aligned}
I^{+} & =\frac{J^{+}}{2} \cdot \frac{\sqrt{s} J^{+} x^{\prime \prime}-2\left(\bar{q}_{1} \cdot\left(\bar{q}_{1}+\bar{q}_{2}\right)\right)+\sqrt{\Lambda^{(2)}}}{\sqrt{s} J^{+} x^{\prime \prime}-\left(\bar{q}_{1}+\bar{q}_{2}\right)^{2}} \\
I^{-} & =\frac{\sqrt{\Lambda^{(2)}}+2\left(\bar{q}_{1} \cdot\left(\bar{q}_{1}+\bar{q}_{2}\right)\right)-\sqrt{s} J^{+} x^{\prime \prime}}{2 J^{+}} \\
Q^{+} & =\frac{J^{+}}{2} \cdot \frac{\sqrt{\Lambda^{(2)}}+2\left(\bar{q}_{2} \cdot\left(\bar{q}_{1}+\bar{q}_{2}\right)\right)-\sqrt{s} J^{+} x^{\prime \prime}}{\sqrt{s} J^{+} x^{\prime \prime}-\left(\bar{q}_{1}+\bar{q}_{2}\right)^{2}} \\
Q^{-} & =\frac{\sqrt{\Lambda^{(2)}}+\sqrt{s} J^{+} x^{\prime \prime}-2\left(\bar{q}_{2} \cdot\left(\bar{q}_{1}+\bar{q}_{2}\right)\right)}{2 J^{+}},
\end{aligned}
$$

while the Mandelstam invariants $\hat{s}_{2}=\left(I^{+}+p^{\prime \prime}\right)^{2}, \hat{t}_{2}=(J-I)^{2}, \hat{u}_{2}=(I-Q)^{2}$ are given by:

$$
\begin{aligned}
& \hat{s}_{2}=\sqrt{s} x^{\prime \prime} I^{+} \\
& \hat{t}_{2}=-\frac{\left(\left(\bar{q}_{1}+\bar{q}_{2}\right) I^{+}-J^{+} \bar{q}_{1}\right)^{2}}{I^{+} J^{+}} \\
& \hat{u}_{2}=\frac{\left(I^{+} \bar{q}_{2}+Q^{+} \bar{q}_{1}\right)^{2}}{I^{+} Q^{+}}
\end{aligned}
$$

By solving (6) with respect to $x$ and $P$ as a function of $x^{\prime}, I$ and $\bar{q}_{1}$ one obtains:

$$
\begin{aligned}
x & =\frac{x^{\prime} I^{+}}{\sqrt{s} x^{\prime}-I^{-}} \\
P^{+} & =\frac{\bar{q}_{1}^{2}}{\sqrt{s} x^{\prime}-I^{-}} \\
P^{-} & =\sqrt{s} x^{\prime}-I^{-}
\end{aligned}
$$


The lower limit of $x^{\prime}$ is derived when requiring $x$ to be smaller than 1 :

$$
x^{\prime} \geq x_{\min }^{\prime} \equiv \frac{I^{-}}{\sqrt{s}-I^{+}}
$$

so we introduce

$$
\Phi^{(1)}=1-\frac{I^{-}}{x^{\prime}\left(\sqrt{s}-I^{+}\right)}
$$

while the expression of the Mandelstam invariants $\hat{s}_{1}=(h+p)^{2}, \hat{t}_{1}=(I-h)^{2}$ and $\hat{u}_{1}=$ $(P-h)^{2}$ is:

$$
\begin{aligned}
& \hat{s}_{1}=s x x^{\prime} \\
& \hat{t}_{1}=-\sqrt{s} x^{\prime} I^{+} \\
& \hat{u}_{1}=-\sqrt{s} x P^{-}
\end{aligned}
$$

[1] A. Accardi et al., arXiv:hep-ph/0308248.

[2] D. Antreasyan, J. W. Cronin, H. J. Frisch, M. J. Shochet, L. Kluberg, P. A. Piroue and R. L. Sumner, Phys. Rev. D 19, 764 (1979).

[3] P. B. Straub et al., 38.8-Gev," Phys. Rev. Lett. 68, 452 (1992).

[4] M. Gyulassy and P. Levai, Phys. Lett. B 442, 1 (1998) [arXiv:hep-ph/9807247].

[5] X. N. Wang, Phys. Rev. C 61, 064910 (2000) [arXiv:nucl-th/9812021].

[6] E. Wang and X. N. Wang, Phys. Rev. C 64, 034901 (2001) [arXiv:nucl-th/0104031].

[7] Y. Zhang, G. Fai, G. Papp, G. G. Barnafoldi and P. Levai, Phys. Rev. C 65, 034903 (2002) [arXiv:hep-ph/0109233].

[8] B. Z. Kopeliovich, J. Nemchik, A. Schafer and A. V. Tarasov, Phys. Rev. Lett. 88, 232303 (2002) [arXiv:hep-ph/0201010].

[9] M. A. Braun, E. G. Ferreiro, C. Pajares and D. Treleani, Nucl. Phys. A 723, 249 (2003) [arXiv:hep$\mathrm{ph} /$ 0207303].

[10] A. Accardi and M. Gyulassy, arXiv:nucl-th/0308029.

[11] S. S. Adler et al. [PHENIX Collaboration], Phys. Rev. Lett. 91, 072303 (2003) [arXiv:nuclex/0306021].

[12] I. Arsene et al. [BRAHMS Collaboration], Phys. Rev. Lett. 91, 072305 (2003) [arXiv:nuclex/0307003].

[13] B. B. Back et al. [PHOBOS Collaboration], Phys. Rev. Lett. 91, 072302 (2003) [arXiv:nuclex/0306025].

[14] J. Adams et al. [STAR Collaboration], Phys. Rev. Lett. 91, 072304 (2003) [arXiv:nucl-ex/0306024]. 
[15] A. Accardi and M. Gyulassy, Proc. 19th Winter Workshop on Nuclear Dynamics (2003).

[16] A. Accardi and D. Treleani, Phys. Rev. D 64, 116004 (2001) [arXiv:hep-ph/0106306].

[17] V. M. Braun and Y. M. Shabelski, Int. J. Mod. Phys. A 3, 2417 (1988).

[18] A. Accardi and D. Treleani, Phys. Rev. D 63, 116002 (2001) [arXiv:hep-ph/0009234].

[19] V. Abramovskii, V.N. Gribov and O.V. Kancheli, Yad. Fiz. 18, 595 (1973) [Sov. J. Nucl. Phys. 18, 308 (1974)].

[20] Y. V. Kovchegov and A. H. Mueller, Nucl. Phys. B 529, 451 (1998) [arXiv:hep-ph/9802440].

[21] K. J. Eskola and H. Honkanen,arXiv:hep-ph/0205048.

[22] H. Lai et al. [CTEQ Collaboration], Eur. Phys. J. C 12 (2000) 375.

[23] A. Accardi et all, arXiv:hep-ph0308248.

[24] B. A. Kniehl, G. Kramer and B. Potter, Nucl. Phys. B 582 (2000) 514.

[25] K. J. Eskola, K. Kajantie, and J. Lindsford, Nucl. Phys. B 323 (1989) 37.

[26] http://www.phenix.bnl.gov/conferences.html

[27] http://www-rnc.lbl.gov/qm2004/talks/plenary/01Monday/MMurray.pdf 


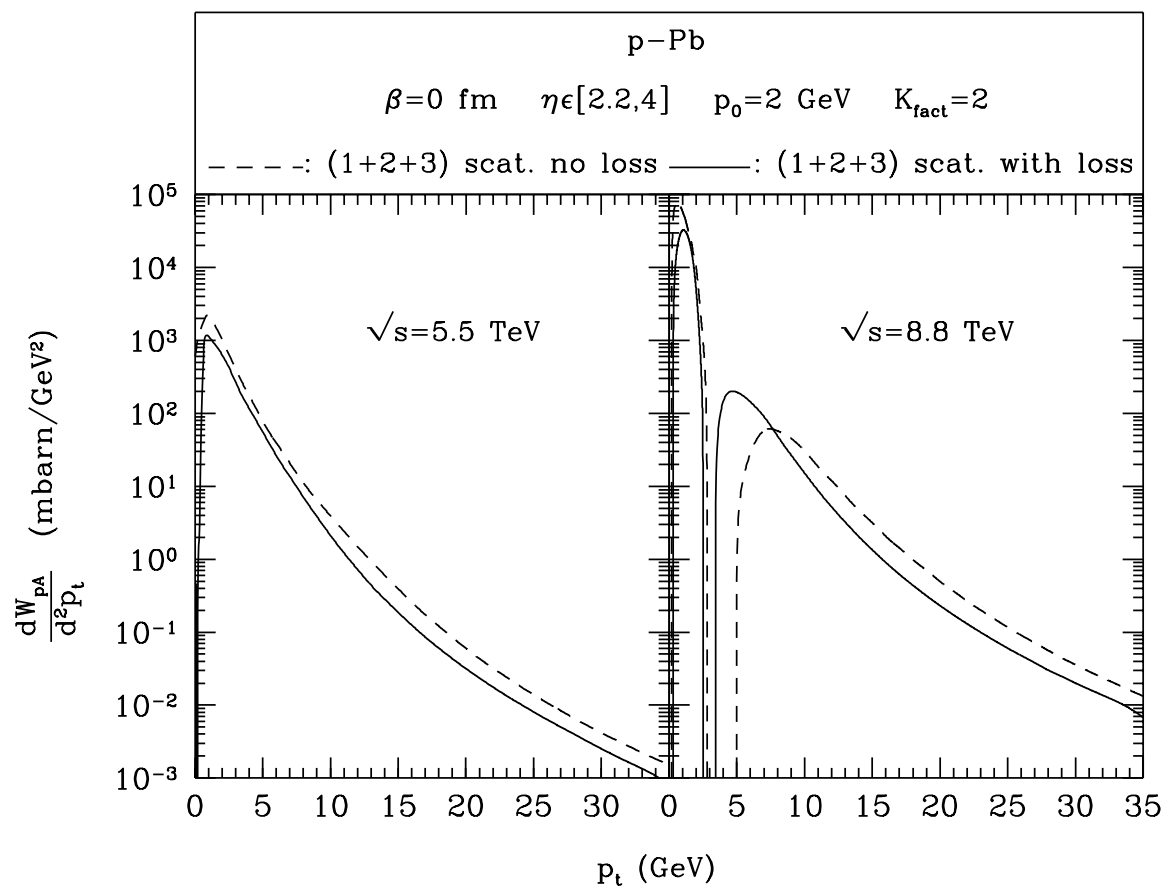

Figure 1: Transverse momentum spectrum of partons produced in $p+P b$ collisions at $\sqrt{s}=$ 5.5, 8.8 ATeV and $\eta \in[2.4,4]$, using $p_{0}=2 \mathrm{GeV}$, and $k_{\text {factor }}=2$. The solid lines are the result of implementing exact kinematics in the calculation, the dashed lines refer to the case of approximate kinematics. 


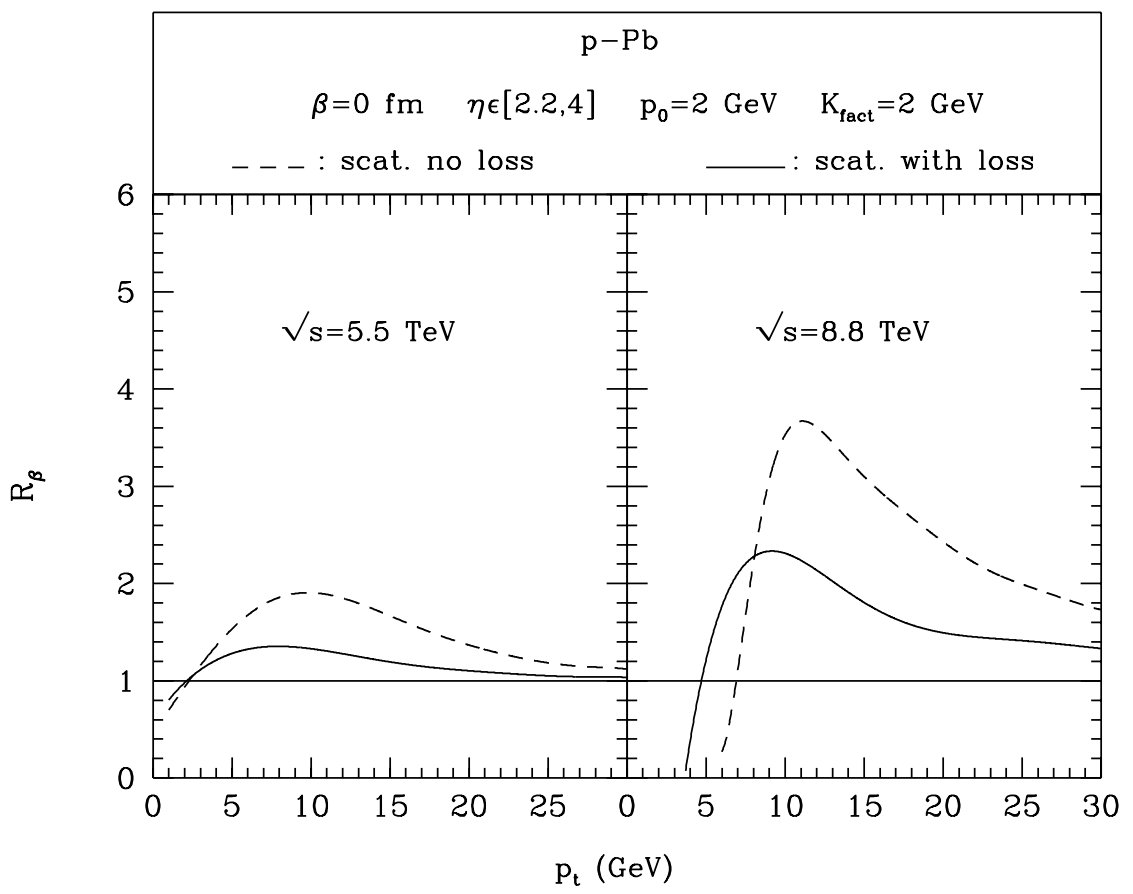

Figure 2: Cronin Ratio in the case of exact kinematics (solid line) and in the case of approximate kinematics (dashed line) with the two different choices of energy scale. 

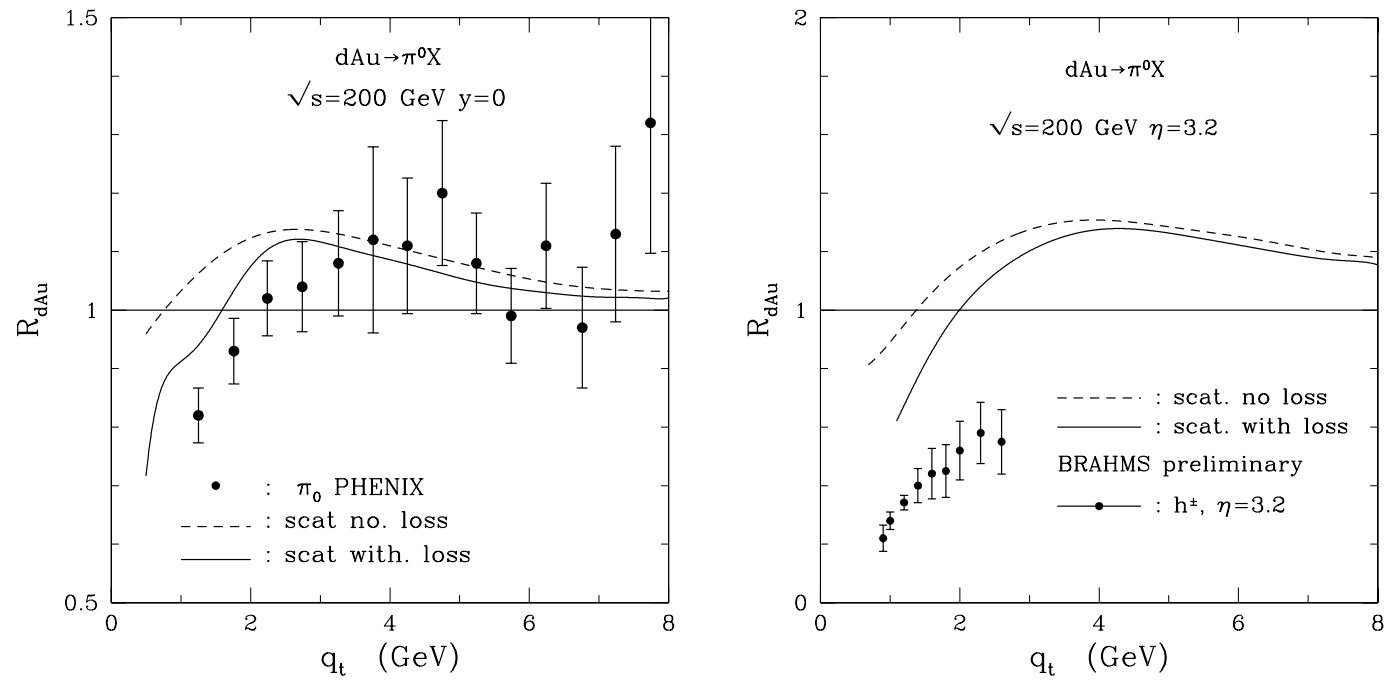

Figure 3: Cronin ratio in $d+A u \rightarrow \pi^{0} X$ collisions at $\sqrt{s}=200 \mathrm{GeV}$. The solid line refers to the case of exact kinematics and the dashed line to the case of approximate kinematics. Left and right panels refer respectively to $y=0$ and $\eta=3.2$. The data are form ref. [11] and [27]. 


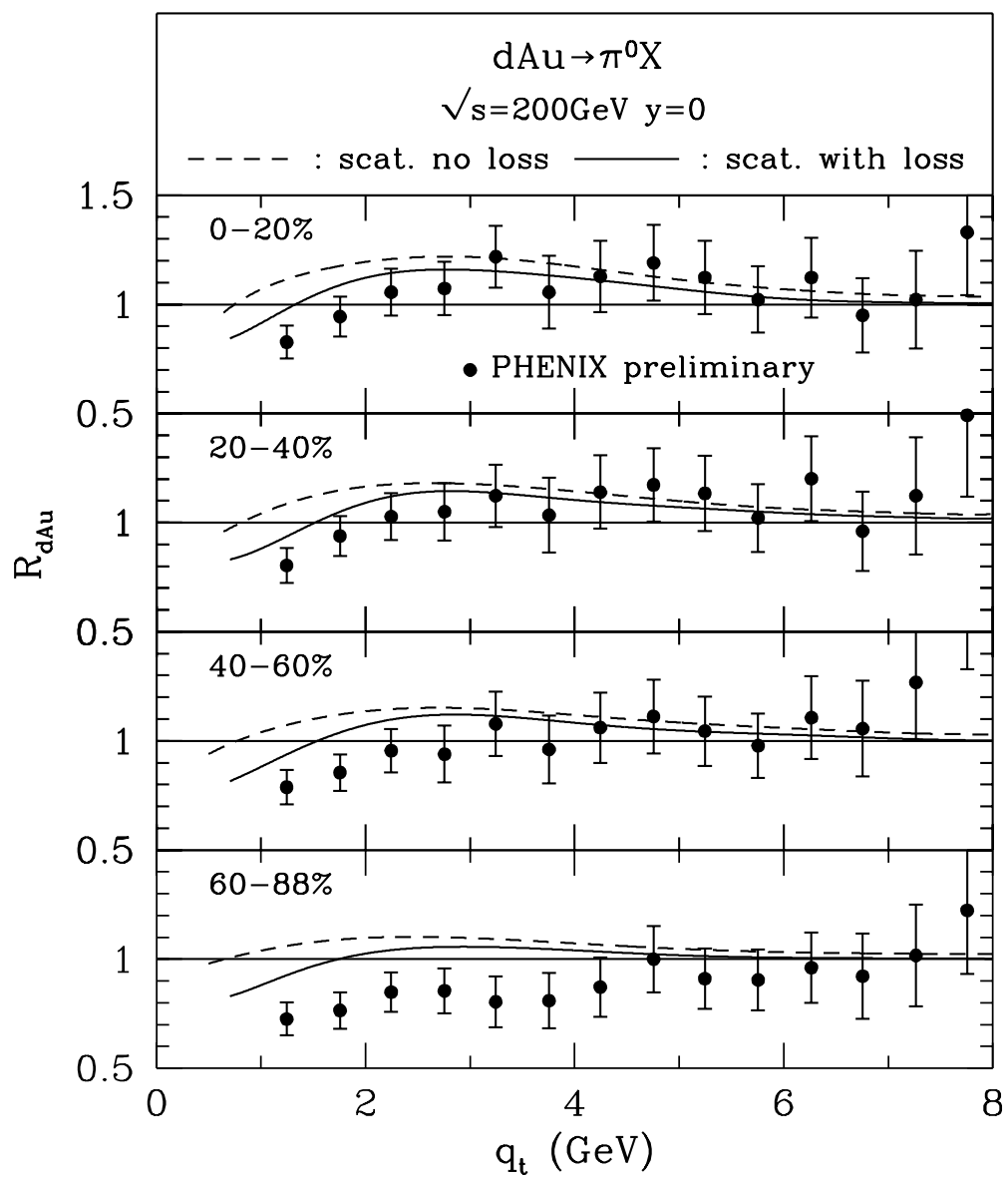

Figure 4: Centrality dependence of Cronin ratio with and without energy loss implementation. Comparison with experimental data presented by PHENIX. 